\title{
Tailored keyhole surgery for basal ganglia cavernous malformation with preoperative three-dimensional pyramidal tracts assessment and intraoperative electrophysiological monitoring
}

\author{
Kai Quan, Geng Xu, Fan Zhao and Wei Zhu*
}

\begin{abstract}
Background: Accurately mapping the pyramidal tracts preoperatively and intraoperatively is the primary concern when operating on cavernous malformations (CMS) in the basal ganglia. We have conducted new methods for preoperative planning and have tailored lesion resection to prevent the damage of pyramidal tracts.

Patients and methods: Eleven patients harboring cavernous malformations in basal ganglia were treated surgically from April 2008 to January 2015. Surgical planning was based on three-dimensional diffusion tensor pyramidal tractography and Virtual Reality system. Intraoperative detecting of pyramidal tracts with subcortical stimulation mapping and motor evoked potential monitoring were performed. The extent of resection and postoperative neurological function were assessed in each case.

Results: Total removal of the cavernous malformations were achieved in each case. Four of the total eleven cases presented temporary neurological deficits, including one occurrence of hemiparesis and three occurrences of hemianesthesia. No permanent neurological deficit was developed in this series of cases.

Conclusion: Three-dimensional diffusion tensor pyramidal tractography is quite helpful for preoperative planning of basal ganglia cavernous malformations, especially in choosing a suitable surgical approach. Intraoperative detection of pyramidal tracts with subcortical stimulation mapping and motor evoked potential monitoring play important roles in preventing damage to pyramidal tracts during lesion resection.
\end{abstract}

Keywords: Cavernous malformation, Keyhole surgery, Diffusion tensor tractography, Intraoperative electrophysiological monitoring

\section{Background}

Surgical removal of basal ganglia cavernous malformations is technically challenging $[1,2]$. Although the introduction and further development of image-guided neurosurgical system has greatly facilitated the challenges encountered in approaching the lesion [3, 4], the high risk of paresis due to damage to the perilesional pyramidal tracts during lesion resection continues to make this procedure controversial [1, 5]. Accurate

\footnotetext{
* Correspondence: drweizhu@sina.com

Department of Neurosurgery, Huashan Hospital, Fudan University, No. 12 Wulumuqi Zhong Road, Shanghai 200040, China
}

assessment of the relationship between the lesion and pyramidal tracts is the major concern in preoperative planning. Using morphological and structural imaging alone, this evaluation may be difficult. Diffusion-tensor MRI (DTI) is used as a primary tool to accurately detect the white matter tracts $[6,7]$. After being reconstructed into virtual 3-Dimensional images and merged with T-2 MR images, more information about the relationship between the cavernous malformation and the surrounding pyramidal tracts can be revealed.

Accurately detecting and localizing the pyramidal tracts during lesion dissection is one of the key issues during the 
operation. Electrophysiological monitoring, which includes somatosensory evoked potential (SSEP), motor function evoked potential (MEP), as well as subcortical stimulation mapping, allows early detection of the functional structure to avoid damaging these areas [8-10].

From April 2008 to January 2015, eleven cases of basal ganglia cavernous malformations were surgically treated in our department. The T2 and DTI MRI were routinely performed, reconstructed, and merged into virtual 3-D images. This was used as the main method for preoperative planning and intraoperative imaging guidance. Somatosensory evoked potential (SSEP), motor function evoked potential (MEP) as well as subcortical stimulation were used intraoperatively. These strategies and techniques are reviewed in this study.

\section{Patients and methods}

\section{Patient characteristics}

From April 2008 to January 2015, eleven patients (6 male, 5 female) harboring cavernous malformation in basal ganglia were treated in our department. The average age was 40.27 years. All of them presented lesion-related hemorrhages. The patient data and clinical information is presented in Table 1.

\section{Preoperative assessment}

T-2 weighted and DTI MRI were performed on each patient. Each set of individual 3-D MRI and DTI data sets were transferred into the VR system (Dextroscope, Volume Interactions Pte. Ltd.,Singapore) through a compact disc. Volume rendering was constructed to generate a patientspecific 3-D stereoscopic cranium model. To aid in the stereoscopic visualization of the fiber tracts, we used the Fiber Tracking module of the VR system. Pyramidal tracts were primarily displayed in blue. Segmentation tools were great aids to extract the $\mathrm{CM}$ from the brain tissue on 3-D MRI, which enabled operators to observe the spatial relationship between the lesion and other components. Fiber tracking and segmentation of the $\mathrm{CM}$ were performed to acquire better understanding of anatomical relationship between the lesions and tracts. The operator visualized the anatomical relationship between the lesion and pyramidal tracts, designed various surgical approaches virtually by using different tools (e.g. picking, cropping, cutting, drilling, etc.). In the end, an optimal patient-specific plan was determined.

Depending on the CM location in relation to the pyramidal tracts, either the trans-frontal anterior-lateral approach or the trans-parietal posterior-lateral keyhole approach was used. The trans-frontal anterior-lateral approach was chosen when the $\mathrm{CM}$ was located anteriorly to the pyramidal tracts, while the trans-parietal posteriorlateral keyhole approach was used when the $\mathrm{CM}$ was located posteriorly to the pyramidal tracts. The patient with the CM protruding to the insular cortex was accessed via the transsylvian approach.

\section{Surgical considerations and techniques Surgical approaches}

Trans-frontal anterior-lateral keyhole approach The patient was placed in supine position with their head slightly rotated contralaterally. A 5-cm straight skin incision and a 3-cm diameter bone window anterior to the coronary fissure was made. After opening the dura, the lesion can be accessed through a trans-sulcus parenchymal corridor with the guidance of neuronavigation. Trans-parietal posterior-lateral keyhole started with a 5$\mathrm{cm}$ straight parietal skin incision when the patient was

Table 1 Patient characteristics and operation surgical procedures

\begin{tabular}{|c|c|c|c|c|c|c|c|c|}
\hline \multirow[t]{2}{*}{$\overline{\text { Patient }}$} & \multirow[t]{2}{*}{ Age/Sex } & \multirow[t]{2}{*}{ presentation } & \multirow[t]{2}{*}{ Size of $\mathrm{CM}(\mathrm{cm})$} & \multirow[t]{2}{*}{ Lesion Location To PT } & \multirow[t]{2}{*}{ Surgical approach } & \multicolumn{3}{|c|}{$\begin{array}{l}\text { Changes of intraoperative electrophysilogica } \\
\text { monitoring }\end{array}$} \\
\hline & & & & & & $\overline{S C S M}$ & SSEP & MEP \\
\hline 1 & $35 / F$ & ICH, epilepsy & 1.5 & Anterior & $A-L$ & + & - & - \\
\hline 2 & $28 / F$ & $\mathrm{ICH}$ & 1.2 & Anterior & A & + & - & - \\
\hline 3 & $38 / F$ & ICH, epilepsy & 1.0 & Posterior-Lateral & P-L & + & - & - \\
\hline 4 & $23 / \mathrm{M}$ & $\mathrm{ICH}$ & 2.0 & Posterior-Lateral & $P-L$ & + & - & - \\
\hline 5 & $43 / \mathrm{M}$ & $\mathrm{ICH}$ & 1.5 & Anterior & A & + & - & - \\
\hline 6 & $49 / M$ & $\mathrm{ICH}$ & 3.5 & Anterior & A & + & - & - \\
\hline 7 & $37 / F$ & $\mathrm{ICH}$ & 4.2 & Anterior & $A-L$ & + & - & - \\
\hline 8 & $34 / \mathrm{M}$ & ICH, epilepsy & 6 & Anterior & $A-L$ & + & - & - \\
\hline 9 & $63 / \mathrm{M}$ & $\mathrm{ICH}$ & 5 & Posterior-Lateral & $P-L$ & + & - & - \\
\hline 10 & $56 / M$ & $\mathrm{ICH}$ & 4 & Anterior & Trans-sylvian & + & - & - \\
\hline 11 & $37 / F$ & ICH, epilepsy & 1.5 & Posterior-Lateral & P-L & + & - & - \\
\hline
\end{tabular}


placed in a lateral position. If the nidus was located near the insular lobe surface, we chose the transsylvian approach. The bone window and lesion exposure were carried out in a similar fashion as the trans-frontal anterior-lateral approach.

Intraoperative monitoring and lesion resection Continous SSEP and MEP monitoring started after the lesion was accessed and subcortical stimulation of the perilesional parenchyma was carried out before dissection. The dissection of the $\mathrm{CM}$ started from a noneloquent plane of cleavage where the stimulation shows negative reaction. Subcortical stimulation was used frequently when suspected pyramidal tract regions were encountered. The part of the lesion adhering to the pyramidal tracts was dissected and separated at the last procedure. Continuous MEP and SSEP monitoring was routinely performed during the lesion resection.

Postoperative evaluation and follow-up Surgical results for all patients were evaluated by T-2 weight DTI MRI within 3 days postoperatively. Clinical outcomes were evaluated immediately after surgery, at the time of discharge, and muscle strength grading and Glasgow Outcome Scale (GOS) were assessed 3 months postoperatively. The data and clinical information is presented in Table 2.

\section{Result}

\section{Clinical outcome}

The trans-frontal anterior-lateral approach was performed in six cases, the trans-parietal posterior-lateral in four, and the transsylvian approach in one case. Three of the total eleven patients presented new postoperative deficits. Temporary hemiparesis occurred in one case but recovered completely within 28 days after surgery. Temporary hemianesthesia occurred in three cases, in

Table 2 Postoperative results

\begin{tabular}{|c|c|c|c|c|c|}
\hline \multirow[t]{2}{*}{ Patient } & \multicolumn{2}{|l|}{ Deficits } & \multirow[t]{2}{*}{ GOS } & \multirow[t]{2}{*}{ Recurrence } & \multirow[t]{2}{*}{ Period of follow-up } \\
\hline & Temporary & Permanent & & & \\
\hline 1 & + & - & 5 & No & $24 \mathrm{~m}$ \\
\hline 2 & + & - & 5 & No & $26 \mathrm{~m}$ \\
\hline 3 & - & - & 5 & No & $11 \mathrm{~m}$ \\
\hline 4 & - & - & 5 & No & $16 \mathrm{~m}$ \\
\hline 5 & + & - & 5 & No & $19 \mathrm{~m}$ \\
\hline 6 & - & - & 5 & No & $12 \mathrm{~m}$ \\
\hline 7 & + & - & 5 & No & $14 \mathrm{~m}$ \\
\hline 8 & - & - & 5 & No & $10 \mathrm{~m}$ \\
\hline 9 & + & - & 5 & No & $11 \mathrm{~m}$ \\
\hline 10 & - & - & 5 & No & $8 \mathrm{~m}$ \\
\hline 11 & - & - & 5 & No & $5 \mathrm{~m}$ \\
\hline
\end{tabular}

GOS glascow outcome score which two were disappeared at 3-months follow-up, and the other one was released at 6-months. No secondary epilepsy was developed postoperatively. The final GOS was 5 in all the patients at the follow-up period of 5-26 months. There was no mortality in this group of patients (Table 1).

\section{Radiological outcome and recurrence}

Intraoperative inspection and postoperative MRI confirmed total removal of the lesion in all patients. No patient presented a recurrence during a mean follow-up of 12.5 months.

\section{Illustrative case 1}

A 35-year-old female patient suffered from refractory epilepsy, which could not be controlled with medication. $\mathrm{CT}$ and MR revealed a CM with mild hemorrhage locating in left basal ganglia. Preoperative assessment with merged 3- Dimensional T-2 weighted and DTI MRI in Virtual Reality showed a CM located anterior and lateral to the pyramidal tracts. CM resection was performed via a trans-frontal lateral keyhole approach. Continuous SSEP, MEP and subcortical stimulation were performed during the lesion dissection and resection to prevent damage to the pyramidal tracts. The lesion was totally removed without any changes of SSEP and MEP monitoring. The patient presented transient weakness of right lower extremity which was completely recovered in two weeks after surgery (Fig. 1).

\section{Illustrative case 2}

A 23-year-old male patient presented with sudden weakness of the left extremities three weeks before surgery. $\mathrm{CT}$ revealed a hemorrhagic lesion located in right basal ganglia. The diagnosis of a CM was confirmed by MRI. Preoperative assessment with merged 3- Dimensional T2 weighted and DTI MRI in Virtual Reality showed a $\mathrm{CM}$ located posterior to the pyramidal tracts. CM resection was performed via a trans-parietal lateral keyhole approach. Continuous SSEP, MEP, and subcortical stimulation were performed during the lesion dissection and resection to prevent damage to the pyramidal tracts. The lesion was totally removed and the DVA located medially was preserved. No change in SSEP or MEP was found during the surgery. The patient did not present any neurological deficit, but had pneumonia. He was treated with antibiotics and completed recovered in 10 days (Fig. 2).

\section{Discussion}

Unlike cerebral arteriovenous malformations, CM rarely cause severe intracranial hemorrhage [11]. However, the incidence of symptomatic recurrent hemorrhage is relatively high [12]. Several authors hold that deep-seated 

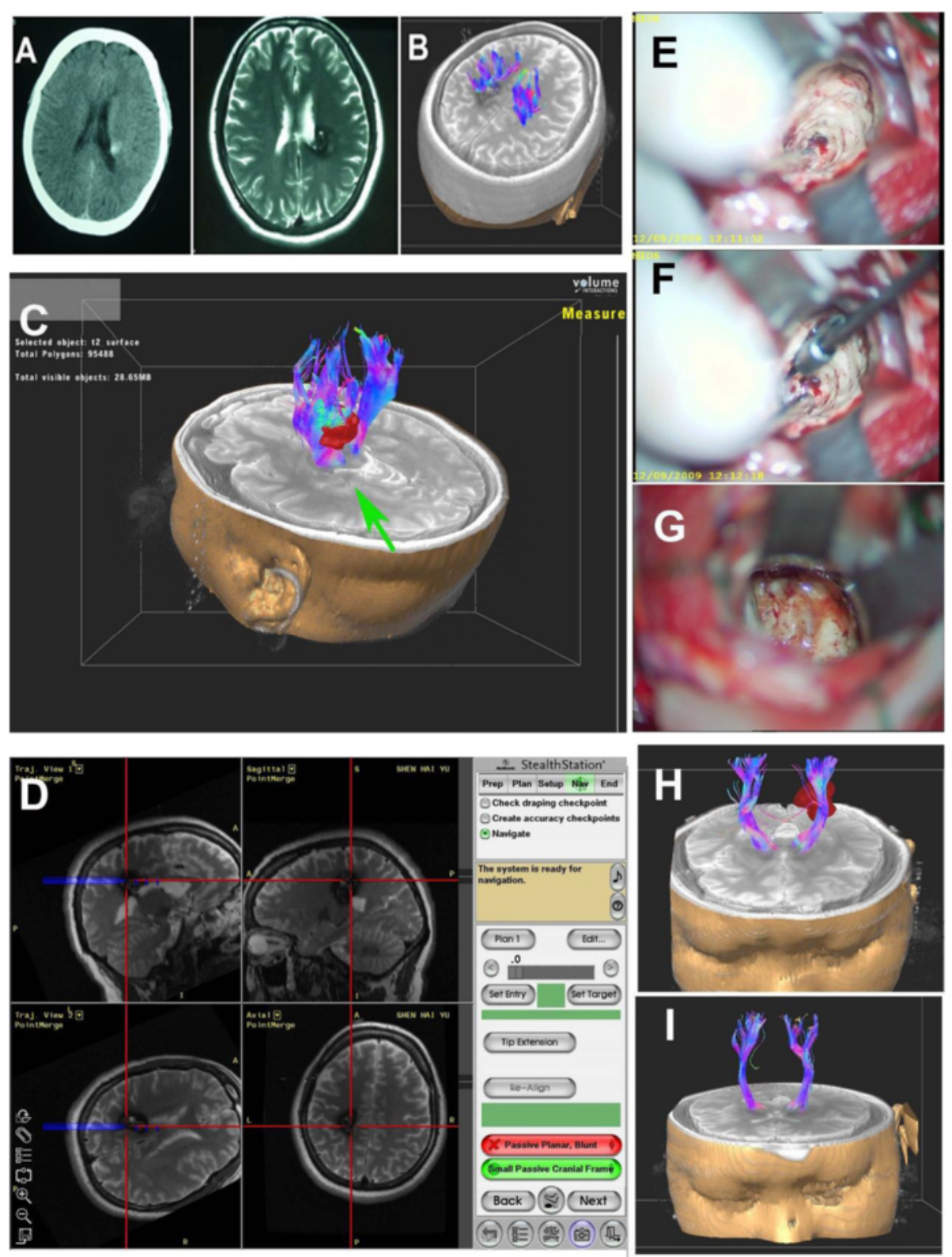

Fig. $1 \mathrm{CT}$ and MRI showed a CM located in left basal ganglia (a). Diffusion tensor tractography delineated pyramidal tracts and combined with T2 MR Image (b). After assessing the relationship between pyramidal tracts and CM via Virtual Reality, posterior lateral approach was chosen as shown by green arrow (c). CM was accessed through posterior lateral approach with the guidance of neuronavigation (d). Intraoperative images showed lesion exposure (e), subcortical stimulation mapping for pyramidal tracts in perilesional region (f), and total removal of CM (g). Preoperative VR image showed CM close to the pyramidal tracts (h), postoperative VR images showed total removal of $\mathrm{Cm}$ without damage to the pyramidal tracts (i)

CMs have a higher rate of hemorrhage and rebleed than superficial CMs [13]. The efficiency of radiosurgery for these lesions is controversial [14]. Radical surgical excision of the lesion is a unique way to eliminate the risk of subsequent bleeding and reduce the symptoms caused by the mass effect. However, due to their critical location, CMs in the basal ganglia present a challenge for the neurosurgeon in term of operative morbidity. While, patients with symptomatic hemorrhage have a very low morbidity in the majority of cases because the CMs can be resected in hemosiderin platform without damaging many the brain tissues.

The surgical indication of the basal ganglia CM in this study are the symptomatic patients who have suffered prior hemorrhages. Surgical planning depends on the accurate imaging and assessment of the relationship between the lesion and the perilesional pyramidal tracts. To visualize the pyramidal tracts, DTI MRI was 

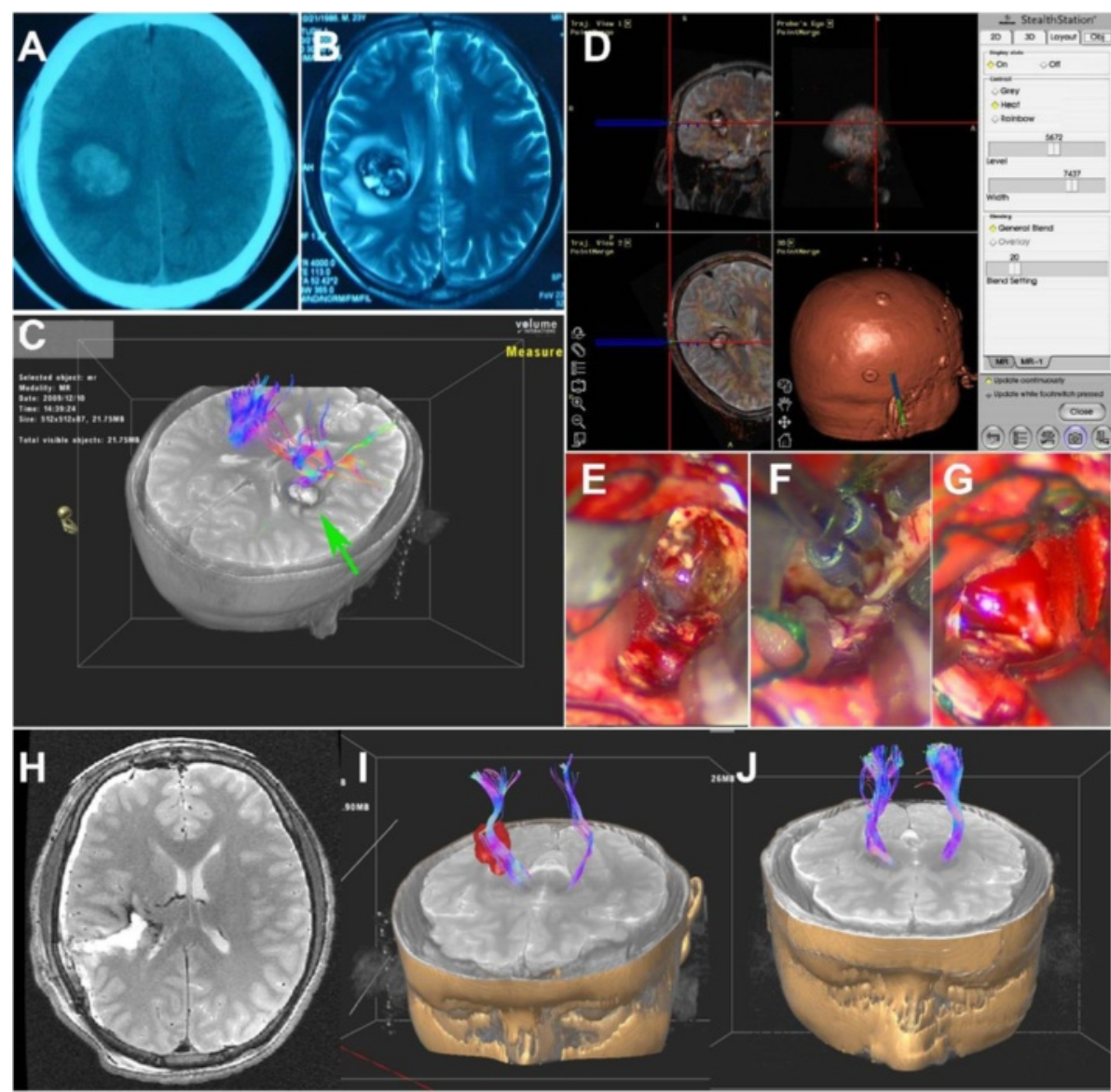

Fig. 2 CT (a) and MR (b) showed a CM located in right basal ganglia. After assessing the relationship between pyramidal tracts and CM via Virtual Reality, posterior lateral approach was chosen as shown by green arrow (c). CM was accessed through posterior lateral approach with the guidance of neuronavigation (d). Intraoperative images showed lesion exposure (e), subcortical stimulation mapping for pyramidal tracts in perilesional region ( $\mathbf{f}$ ), and total removal of CM (g). Postoperative MR (T2 weighted) showed CM was totally removed (h). Preoperative VR image showed CM close to the pyramidal tracts (i), postoperative VR images showed total removal of $\mathrm{Cm}$ without damage to the pyramidal tracts (j)

performed and reconstructed into a 3-D fashion. After being merged with T-2 images of the lesion in Virtual Reality, the lesion location in relation to the pyramidal tracts could be assessed clearly. Virtual Reality also allowed the operator to simulate the surgical procedures. The key in planning the surgical approach is to avoid traversing the pyramidal tracts before exposing the CM. Typically approaches to the basal ganglia have included the lateral approach, the transsylvian approach, and the supracarotid triangle approach [15]. Other authors have used contralateral interhemispheric approach to deepseated periventricular cavernous malformations [16], we prefer using either an anterior or posterior lateral approach. We approach the lesion through a small sulcus incision and parenchyma corridor after opening a keyhole bone window. The pathways through the anterior insula, mediobasal frontal lobe, and anterior perforated substance cannot be considered as completely noneloquent trajectories [17], Continuous SSEP and MEP were performed during the lesion dissection and resection. No long-term approach-related complication will result since no eloquent structure is dissected. We usually do not propose the transsylvian-transinsular approach unless the CM protrudes to the insular cortex. The manipulation of lenticulostriate arteries during dissection of the sylvian fissure and insular parenchyma could lead to severe postoperative neurological deficits. The brain shift caused by excessive CSF drainage during sylvian fissure dissection make the localization of the lesion more difficult.

Early detection of the pyramidal tracts that are possibly involved in or near the lesion is the key to their preservation. We used subcortical stimulation mapping as the real-time motor function testing to identify the pyramidal tracts [18]. We usually stimulated the perilesional parenchyma before the CM resection. The dissection of the $\mathrm{CM}$ started from a noneloquent plane of cleavage where the stimulation showed negative reaction. 
It was necessary to limit the resection to the $\mathrm{CM}$ while the hemosiderin-stained brain was left intact to avoid damage to viable structures. After fresh or subacute hematoma was removed and the $\mathrm{CM}$ was resected in a piece-meal like excision, the lesion was internally decompressed. The interface between the CM and the eloquent perilesional parenchyma was identified by stimulation as pyramidal tracts region was clearly visualized after most part of the lesion was resected and larger surgical cavity was formed. Finally, the residual lesion, which adheres to the pyramidal tracts, could be easily dissected with no pressure to the tracts. Continuous MEP and SSEP monitoring were performed during the lesion resection. Any changes in MEP or SSEP indicated that potential damage to the motor tracts would result if surgical manipulation in that region continuous. The operator would stop the operations in that region until the MEP and SSEP returned to normal. The manipulation in a safer area continuous until the CM was totally removed. Meticulous inspection of the lesion bed under high magnification is necessary to identify residual lesion.

The bleeding in surgical cavity can usually be controlled by gentle pressure and local application of a hemostatic agent instead of cauterization. The preservation of the developmental venous anormalis (DVAs) during or after $\mathrm{CM}$ resection is recommended to prevent postoperative severe brain edema or hemorrhagic infarction, which could lead to obvious postoperative neurological deficits [19].

Today, most authors advocate early surgery between 3 to 8 weeks of ictus. Similarly, we prefer to remove the hemorrhagic $\mathrm{CM}$ in basal ganglia during the subcute phase, usually within $2-4$ weeks $[20,21]$. A plane of cleavage can be easily found in this period. A delayed surgery will allow pseudocapsule formation in the surrounding parenchyma, and the lesion will adhere to the gliotic and hemosiderin-stained brain more tightly, making it even more difficult to totally resect the lesion without any injury to the pyramidal tracts.

\section{Conclusion}

Three-dimensional diffusion tensor pyramidal tractography is quite helpful for preoperative planning of basal ganglia cavernous malformation, especially in choosing a suitable surgical approach. Intraoperative detecting of pyramidal tracts with subcortical stimulation mapping, motor evoked potential monitoring play important roles in preventing damage to pyramidal tracts during lesion resection.

\section{Consent}

Informed consent was obtained from all patients for the publication of this report and any accompanying images.

\section{Competing interests}

The authors have no personal financial or institutional interest in any of the drugs, materials, or devices described in this article.

\section{Authors' contributions}

WZ conceived the project and designed the study. KQ collected patient's data. FZ provided technical assistance in the study. GX prepared the illustration. KQ analyzed data and wrote the paper. All authors approved the paper for the submission.

\section{Acknowledgements}

The authors sincerely thank Miss Angie Zou for editorial assistance, and this work was supported by "Science Funding of shanghai municipal commission of Health and Family Planning" grant XYQ2011019 (to Wei Zhu).

Received: 21 July 2015 Accepted: 29 September 2015

Published online: 18 December 2015

\section{References}

1. Gross BA, Batjer HH, Awad IA, Bendok BR. Cavernous malformations of the basal ganglia and thalamus. Neurosurgery. 2009;65(1):7-18. discussion 18-19.

2. Gralla J, Ganslandt O, Kober H, Buchfelder M, Fahlbusch R, Nimsky C. Image-guided removal of supratentorial cavernomas in critical brain areas: application of neuronavigation and intraoperative magnetic resonance imaging. Minim Invasive Neurosurg. 2003;46(2):72-7.

3. Winkler D, Lindner D, Strauss G, Richter A, Schober R, Meixensberger J. Surgery of cavernous malformations with and without navigational support-a comparative study. Minim Invasive Neurosurg. 2006;49(1):15-9.

4. Mao Y, Zhou L, Du G, Chen L. Image-guided resection of cerebral cavernous malformations. Chin Med J. 2003:116(10):1480-3.

5. Kim MS, Pyo SY, Jeong YG, Lee SI, Jung YT, Sim JH. Gamma knife surgery for intracranial cavernous hemangioma. J Neurosurg. 2005;102(Suppl):102-6.

6. Chen X, Weigel D, Ganslandt O, Fahlbusch R, Buchfelder M, Nimsky C. Diffusion tensor-based fiber tracking and intraoperative neuronavigation for the resection of a brainstem cavernous angioma. Surg Neurol. 2007;68(3):285-91. discussion 291.

7. Chen X, Weigel D, Ganslandt O, Buchfelder M, Nimsky C. Diffusion tensor imaging and white matter tractography in patients with brainstem lesions. Acta Neurochir. 2007;149(11):1117-31. discussion 1131.

8. Okada T, Mikuni N, Miki Y, Kikuta K, Urayama S, Hanakawa T, et al. Corticospinal tract localization: integration of diffusion-tensor tractography at 3-T MR imaging with intraoperative white matter stimulation mappingpreliminary results. Radiology. 2006;240(3):849-57.

9. Mikuni N, Okada T, Nishida N, Taki J, Enatsu R, Ikeda A, et al. Comparison between motor evoked potential recording and fiber tracking for estimating pyramidal tracts near brain tumors. J Neurosurg. 2007;106(1):128-33.

10. Kamada K, Todo T, Ota T, Ino K, Masutani Y, Aoki S, et al. The motor-evoked potential threshold evaluated by tractography and electrical stimulation. J Neurosurg. 2009;111(4):785-95.

11. Batra S, Lin D, Recinos PF, Zhang J, Rigamonti D. Cavernous malformations: natural history, diagnosis and treatment. Nat Rev Neurol. 2009:5(12):659-70.

12. Hauck EF, Barnett SL, White JA, Samson D. Symptomatic brainstem cavernomas. Neurosurgery. 2009:64(1):61-70. discussion 70-61.

13. Conforto AB, Smid J, Marie SK, Ciriaco JG, Santoro PP, Leite Cda C, et al. Bilateral olivary hypertrophy after unilateral cerebellar infarction. Arq Neuropsiquiatr. 2005;63(2A):321-3.

14. Liscak R, Vladyka V, Simonova G, Vymazal J, Novotny Jr J. Gamma knife surgery of brain cavernous hemangiomas. J Neurosurg. 2005;102(Suppl):207-13.

15. Chang EF, Gabriel RA, Potts MB, Berger MS, Lawton MT. Supratentorial cavernous malformations in eloquent and deep locations: surgical approaches and outcomes. Clinical article. J Neurosurg. 2011:114(3):814-27.

16. Zaidi HA, Chowdhry SA, Nakaji P, Abla AA, Spetzler RF. Contralateral interhemispheric approach to deep-seated cavernous malformations: surgical considerations and clinical outcomes in 31 consecutive cases. Neurosurgery. 2014;75(1):80-6.

17. Abla AA, Spetzler RF, Albuquerque FC. Trans-striatocapsular contralateral interhemispheric resection of anterior inferior basal ganglia cavernous malformation. World Neurosurg. 2013;80(6):e397-9. 
18. Berman Jl, Berger MS, Chung SW, Nagarajan SS, Henry RG. Accuracy of diffusion tensor magnetic resonance imaging tractography assessed using intraoperative subcortical stimulation mapping and magnetic source imaging. J Neurosurg. 2007;107(3):488-94.

19. Perrini $P$, Lanzino $G$. The association of venous developmental anomalies and cavernous malformations: pathophysiological, diagnostic, and surgical considerations. Neurosurg Focus. 2006;21(1):e5

20. Pandey P, Westbroek EM, Gooderham PA, Steinberg GK. Cavernous malformation of brainstem, thalamus, and basal ganglia: a series of 176 patients. Neurosurgery. 2013;72(4):573-89. discussion 588-579.

21. Bruneau M, Bijlenga P, Reverdin A, Rilliet B, Regli L, Villemure JG, et al. Early surgery for brainstem cavernomas. Acta Neurochir. 2006;148(4):405-14.

\section{Submit your next manuscript to BioMed Central and take full advantage of:}

- Convenient online submission

- Thorough peer review

- No space constraints or color figure charges

- Immediate publication on acceptance

- Inclusion in PubMed, CAS, Scopus and Google Scholar

- Research which is freely available for redistribution 